\title{
Molecular cloning and development of RAPD-SCAR markers for Dimocarpus longan variety authentication
}

\author{
Luquan Yang ${ }^{1}$, Shelly Fu², Md Asaduzzaman Khan ${ }^{1}$, Weimin Zeng ${ }^{3^{*}}$ and Junjiang Fu ${ }^{1^{*}}$
}

\begin{abstract}
As an edible fruit and source of traditional medicine, D. longan is grown in most areas of Southern China. Identification of $D$. longan cultivars by using molecular markers is important genetically. In this study, we cloned fragments from improved randomly amplified polymorphic DNA (RAPD), and developed stably diagnostic sequence-characterized amplified region (SCAR) markers. The specific RAPD bands of D. longan cultivars from Guangxi, with size ranging from 500 bp to 900 bp were gel-purified, cloned and sequenced. Four clones named LY2-1, LY4-7, LY4-8 and LY5-2 were identified. In order to investigate whether the fragments were specific for the species, four pairs of SCAR primers were then designed. PCR amplifications were conducted to analyze 18 samples including different $D$. Iongan cultivars and other species. The specific bands with expected sizes were amplified in five $D$. longan samples but not in others. To identify and characterize the difference between $D$. longan and $D$. confinis, PCR amplifications were performed again. The specific bands with expected sizes were found in D. longan but not in D. confinis by SCAR markers LY2-1, LY4-7 and LY5-2, respectively. These results showed that our developed SCAR markers could be very useful as a specific $D$. longan variety authentication. Therefore, our study provides an effective and precise PCR-based diagnostic method and markers to identify D. longan species.
\end{abstract}

Keywords: Dimocarpus longan Lour; Dimocarpus confinis; Improved RAPD; Cloning; SCAR marker; Authentication

\section{Introduction}

Dimocarpus longan Lour (D. longan), also called longan or dragon eye, is a commercially attractive fruit that is widely distributed in subtropical areas. China is the world's largest cultivator of $D$. longan, with more than 2000 years history of cultivation. As a traditional medicine, $D$. longan fruit is used for enhancing memory, promoting blood metabolism, relieving insomnia, and preventing amnesia. Its secondary metabolic products have been also shown to have anti-oxidative, anti-obesity, anti-cancer, anti-tyrosinase, and immunomodulatory activities (Park et al. 2010; Prasad et al. 2010; Zhong et al. 2010). A number of researches have been conducted to give an insight of genetic characterization and authentication in D. longan samples.

\footnotetext{
* Correspondence: zengwmin@yahoo.com.cn; fujunjiang@hotmail.com ${ }^{3}$ Department of Biochemistry, School of Life Sciences, Central South University, Changsha, Hunan 410013, China

${ }^{1}$ Research Center for Preclinical Medicine, Luzhou Medical College, Luzhou, Sichuan 646000, China

Full list of author information is available at the end of the article
}

Several molecular markers have been developed and applied since 1990, including random amplified polymorphic DNA (RAPD) (Williams et al. 1990; Devaiah and Venkatasubramanian, 2008; Chen et al. 2010; Yazbeck et al. 2011; Bhat et al. 2012; Shakeel et al. 2013; Noormohammadi et al. 2013; Zhang et al. 2013), inter-simple sequence repeats (Feofilov et al. 2011; Ganopoulos et al. 2011; Noormohammadi et al. 2013; Zhang et al. 2013), internal transcribed spaces (Varela et al. 2004) and amplified fragment length polymorphism (Vos et al. 1995). These molecular markers have been extensively utilized in various fields for the assessment of genetic diversity, genotype fingerprinting, and molecular breeding.

RAPD markers are DNA fragments from PCR amplification of the genomic DNA's random segments with single primer of arbitrary nucleotide sequence (Williams et al. 1990). It is a relatively easy, inexpensive and rapid technique because of its simplicity and requirement for minimal amounts of genomic DNA (Micheli et al. 1994). 
It has been widely used in the identification and genetic relationship analysis of a number of plant and animal species. The improved RAPD can improve the resolution of the PCR products and its repeatability (Fu et al. 2000; $\mathrm{Fu}$ et al. 2013; Mei et al. 2013). The sequence characterized amplified region (SCAR) marker is one of the stable markers, generally derived from random amplified polymorphic DNA (RAPD) (Dnyaneshwar et al. 2006; Li et al. 2010; Rajesh et al. 2013). The basic principle is to convert the dominant markers into co-dominant markers to reduce the tedious procedures of RAPD (Li et al. 2010; Rajesh et al. 2013). These markers generally reveal higher levels of polymorphism owing to higher annealing temperatures and longer primer sequence specificity (Kumla, et al. 2012). With SCAR marker, analysis is reduced to a simple PCR analysis using PCR primers designed from the sequence of the amplicon of RAPD (Kumla, et al. 2012; Rajesh et al. 2013).

Dimocarpus confinis is a species of plant in the genus Dimocarpus, which is grown in a range of Southern China to Southeast Asia. It is mainly grown as ornamental plants but cannot be used as a food source due to its poisonous nature. D. confinis produces ovalshaped drupe fruits, which is very similar to $D$. longan, causing difficulty in distinguishing it from $D$. longan in the market, if anyone ever sells $D$. confinis as longan [Source: http://www.people.com.cn/GB/paper503/13144/ 1179090.html]. In this study, we aimed to distinguish $D$. longan from $D$. confinis by genetic characterization using RAPD and SCAR. In order to increase the reproducibility and reliability of PCR assays in D. longan and distinguish it from $D$. confinis, SCAR markers have been developed from clones of RAPD fragments. In the present study, the DNA fragments were amplified with the DNA template of $D$. longan from Guangxi Province, using an improved RAPD (Fu et al. 2000; Fu et al. 2013; Mei et al. 2013) followed by DNA ligation, cloning, and sequencing. After a series of experiments, four new specific longan DNA fragments had been confirmed. According to the sequencing results, 4 pairs of primers (SCAR markers) had been designed to converted, which detected specificity of $D$. longan varieties. Three of SCAR markers were useful to distinguish $D$. confinis from D. longan. Therefore, the SCAR primers can be used to assess the genetic diversity and population structure of $D$. longan from $D$. confinis.

\section{Materials and methods}

\section{Genomic DNA extraction}

The DNAs were extracted from fresh young leaves of $D$. longan and $D$. confinis by using previously described slightly modified Cetyl trimethylammonium bromide (CTAB) method (Mei et al. 2013) and stored at $-20^{\circ} \mathrm{C}$ till to use. The fresh young leaves of $D$. confinis were provided by Dr. Jiechun Pan from Agricultural College of Guangxi University in Guangxi Province. Leaves were first fixed in fixing solutions containing chloroform, (without liquid nitrogen), and then grinded into tiny pieces by silica $\left(\mathrm{SiO}_{2}\right)$ for the extraction of DNA with $\mathrm{CTAB}$ method. DNA quality was determined after electrophoresis on $1 \%$ agarose gels. DNA concentration and quality was measured by spectrophotometry at 260 and $280 \mathrm{~nm}$ and normalized to a concentration of $10 \mathrm{ng} / \mu \mathrm{l}$, then stored at $-20^{\circ} \mathrm{C}$ for further study (Mei et al. 2013; Fu, 2012).

\section{Improved RAPD amplification}

The $D$. longan DNAs were initially screened with three random primers (Mei et al. 2013). The PCR reactions were performed with Tiangen reagents (Beijing, China). The improved RAPD reaction solution consisted of $7.5 \mu \mathrm{l} 2 \times \mathrm{Taq}$ PCR MasterMix, $1.5 \mu \mathrm{l} 2.5 \mu \mathrm{M}$ primer and $1.5 \mu \mathrm{l}$ genomic DNA, to a total volume of $15 \mu \mathrm{l}$. Amplification reactions were performed in an Eppendorf Authorized Thermal Cycler (Mastercycler 5331 system, Eppendorf, Germany) under the following program, which involves an initial pre denaturation at $95^{\circ} \mathrm{C}$ for $90 \mathrm{~s}$. It was then followed by 40 cycles of denaturation at $94^{\circ} \mathrm{C}$ for $40 \mathrm{~s}$, annealing at $36^{\circ} \mathrm{C}$ for $60 \mathrm{~s}$, and extension at $72^{\circ} \mathrm{C}$ for $90 \mathrm{~s}$. The final extension step was performed at $72^{\circ} \mathrm{C}$ for $5 \mathrm{~min}$. During the procedure, the temperature rose at the rate of $0.3^{\circ} \mathrm{C} / \mathrm{s}$, and declined at the rate of $3^{\circ} \mathrm{C} / \mathrm{s}$. The amplified products were detected with electrophoresis on $1.5 \%$ agarose gel.

\section{Cloning and sequencing of DNA fragments}

Four different bright bands were excised from agarose gel and purified with TIANgel Midi Purification Kit (DP209, China) according to the company provided protocol. Purified DNA fragments were ligated into pGM-T vector (No. VT202) (Tiangen reagents, Beijing, China), and transformed in DH5 $\alpha$ E. coli complement cells and the recombinant clones were selected on LB agar plates containing $100 \mu \mathrm{g} / \mu \mathrm{l}$ of ampicillin, $40 \mathrm{mg}$ of X-gal and $160 \mu \mathrm{g}$ of IPTG. The blue white screening was

Table 1 Sequences of SCAR primers, PCR condition and product size

\begin{tabular}{|c|c|c|c|c|c|c|}
\hline SCAR & 5'-primer & Sequence $\left(5^{\prime}-3^{\prime}\right)$ & 3'-primer & Sequence $\left(5^{\prime}-3^{\prime}\right)$ & Size (bp) & $\operatorname{Tm}\left({ }^{\circ} \mathrm{C}\right)$ \\
\hline LY2-1 & LY2-1 L & AACTGGAAGTCCCTGGTCCT & LY2-1R & ACAAGAGGCCCCAGTAAGGT & 350 & 60 \\
\hline LY4-7 & LY4-7 L & GGCGCCGGTATACTTTGTAA & LY4-7R & CTCGTAAGAGGATCCGTCCA & 367 & 60 \\
\hline LY4-8 & LY4-8 L & CCCCATCTGGTTGTAGCACT & LY4-8R & AGCCAGCTCAACCAACTCAT & 358 & 60 \\
\hline LY5-2 & LY5-2 L & TITAAATGTGGGCATTTGG & LY5-2R & GCTAACCGAGATCCACTAACG & 250 & 60 \\
\hline
\end{tabular}



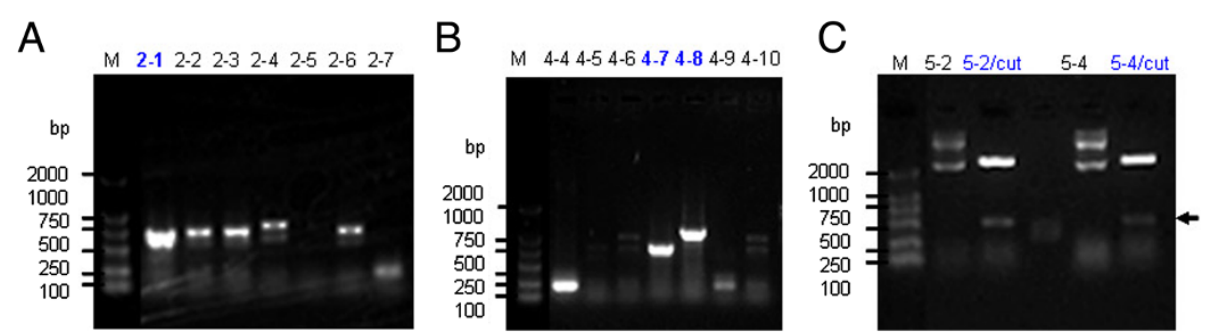

Figure 1 Cloning and identification of positive clones from $D$. longan RAPD products. A. Identification of positive clone 2-1 by PCR amplification with vector T7/sp6 primers. B. Identification of positive clones 4-7 and 4-8 by PCR amplification vector T7/sp6 primers.

C. Identification of positive clones 5-2 and 5-4 by EcoRI digestion with extracted plasmids. Lane " $\mathrm{M}$ " indicates the DNA molecular weight marker DL2000 with the fragment size (bp) 2000, 1000, 750, 500, 250, 100. The arrow indicates the inserted band in the clones 5-2 and 5-4.

A

1 CGOCTAGTCACGACAAA TACTCTGCCCTGGGIATGIGGICTCOCTAGOG GOCTTCTGGATGGGG GOCTCTACCTCCTOC

81 TCTACCACCC TAACTGGAAGTCOCTGGTOC TCTCATT TGCGGACT TCTCCTGITTCA GCCC TCCTCTCTACTAGTTT COC

161 CCTCAGTACTAACTCTGCACT TCOCA TTGITTTCGATCCCTTTCT CCOGACAGICTCTCGTAAAATGICOCTTCTCACCA

241 TAATCA TAACAACC TGI GATA COCAAATCA CACTCTCOCTAATCAAATT TCOCTTCA TTTGCTTTAGGTACAACCTCGGT

321 CTTAGGACTCTCACGGIGGCGAACGGAACOCTGACA CATT GCGGCTTT CСAACTOCGCTGTTTACTACCA.GCCACCTA

401 GACTTGTCTCTAATCCTAGCAACCTTACTGGCCCTCTIGTATCCCATCCTAAACTA TCOCCGTGCTGITGIT TCTGACT 481 AGCOCG

B

1 CTAACCGAAAAAACATA TACTAATOCTTTGACAAAAAAATGCTTCAAAAATCT TGTAATCCTTCAATTGATCAAACT TCA

81 ATGITCAAAAACCTTCAATG ITATTATCA GCAATAAGAAGAAAA GCAAATAT GCACAATCAATGGTAATCAAATCTCA. 161 GAAACCATAAATGGACAAGAATGCAGAAGA GAAAATA TCCA CGATATTGTCAGCAAGAAGAAGGI TAGT TTCGTAATTTT 241 AATGIT CGCCATTT GCGTAATACAAACTTAAGCGTATTTTCGIAATCTT GCGTATATAATGAAAA TAATTTGITTAAGAG 321 GICAAAAAACAAAAATT TTCT TTTTA ITTGGGTAAAA TCTA TAAA TTTCCCTT ITTT TTAA CCAAATTACAAT TCAGTTA. 401 TCCCATTGAAATGATT TACAAGTGGAGTAACTGTTTCCCTTTTA TTACAATTGACCCCOCCTACCGITAGTGGATCTCG 481 GTIACC

C

1 CA TTAG TACGCCACGCACCCC TCCTGACCAAACCAAACCAGCCCGACATATTGACTGTCTA TCTGCCOG TATCOCAACAC 81 GCOCTGAGTGCACCATT CATAAGCCAAGAGGCAAACA GAGC TCAGGCCCCGGTATACTTTGTAG TAAGAACC TGGICCA 161 AGCACAAACCAGGTACAOCOCAATCGAGCAGCTCGOC TACT GICT GGGGATCGCATCCACAAAGC TCCGTOCA TACT TOC 241 AAGCCCATAA GATCGAAGTOC TATCCAGGTATOC TTT GAAGCAAA TOCTCCAGAACCCACA TACC TOCGGIOGACTCATC 321 AAGTGGCOCA TTCACCTCGOCCAATA TCACATCGAGI TCAGACCAAGCCCCGCAATCAAGGGCCAACCTTTCGTACACTT 401 CATCCCTCAGTTTACAGCAOCGCCAGACCA TCAAGTT CAGACTICTCAAGCCCCATACTCGGACC TTTT TGIGGACGGAT 481 OCTCTTACCACACAAGT GOCGCAGCTCCAATOCT GCT CATTAATCCTTACGGTGGIAAGTT TCTT TGTGOCCTACT

D

1 TA GIAGGCCACCOC TCAAATGCTCAT COCT TCCA GTTCATTTTTT CAAACTACATTCACCT ICTT TOOCTTAGCTOC TAT 81 AT TCATTCCA TOCT TATATTAATCACAAGC TAGI TGI TCCT TCOCTCOCATATATCC TATCCCAT TAGGGGIT GCAAATT 161 TCACTA TGAGAGTTCTGGITGAGGIGACCA TATCCAGGTCGTTCA TCCCAGCCTITT TACCACCA TATT GTAACCAGAAG 241 GGAGGTTATCATTACAAAGTCTCCTAATACAATCCACTGTCCACCTCCATCTCCCACAATCAAGGGTAATCTAATT TCC 321 CCAATAAGAGTCACCCAATCT OCAGT GAAACCATACAAAGGTTCAGCGTAAGGCTTCACCT GITTATCTCCAA TCOCCAT 401 CT GIT GTAGCACTCTT TATACATTA TGIT TACCAAGCTACCTATATCAATCA TTATTCTTCTAACCTC TCCA TTOCCAA 481 TACCAGCTTACATAACCAACACATCA TTATCCCGCCAATCAAACCOCT CACA TCTTCTTC TATAAAAA TTATCTCT TCA 561 GA TCOCATCT TTACTCT TTTAGCACA COCT TCTGACTCTCT TCAACCCA TCAGTTCAATATGGTTAGTC TOCCCCACGTA 641 CCGCTCTTACGAOC TGI TTCA GGTACTTCC TATA TCA GIC TOCCATAGAAAG TAAAAATGGTOCTTAT TGTAACCGTTG 721 GI TCTTAAICATCA GTT GIT GACCT GCCT GCTACTGTTGTCCCTGTTGTGGITCAGGTTGAGCA TGATGTTGTAGGAT 801 GGTCGAACCGTAGTCATACTA TATAA TCOC TCAGATGACCA TTTC TAATCATC TTTT TAAT GCCGTCTT TCAA TCOCCAA 881 CA TTCTCACGTGTTGTGCOCTAC

Figure 2 Cloned sequences information by Sanger-sequencing. A. The sequences of clone 2-1. B. The sequences of clone 5-2. C. The sequences of clone 4-7. D. The sequences of clone 4-8. 


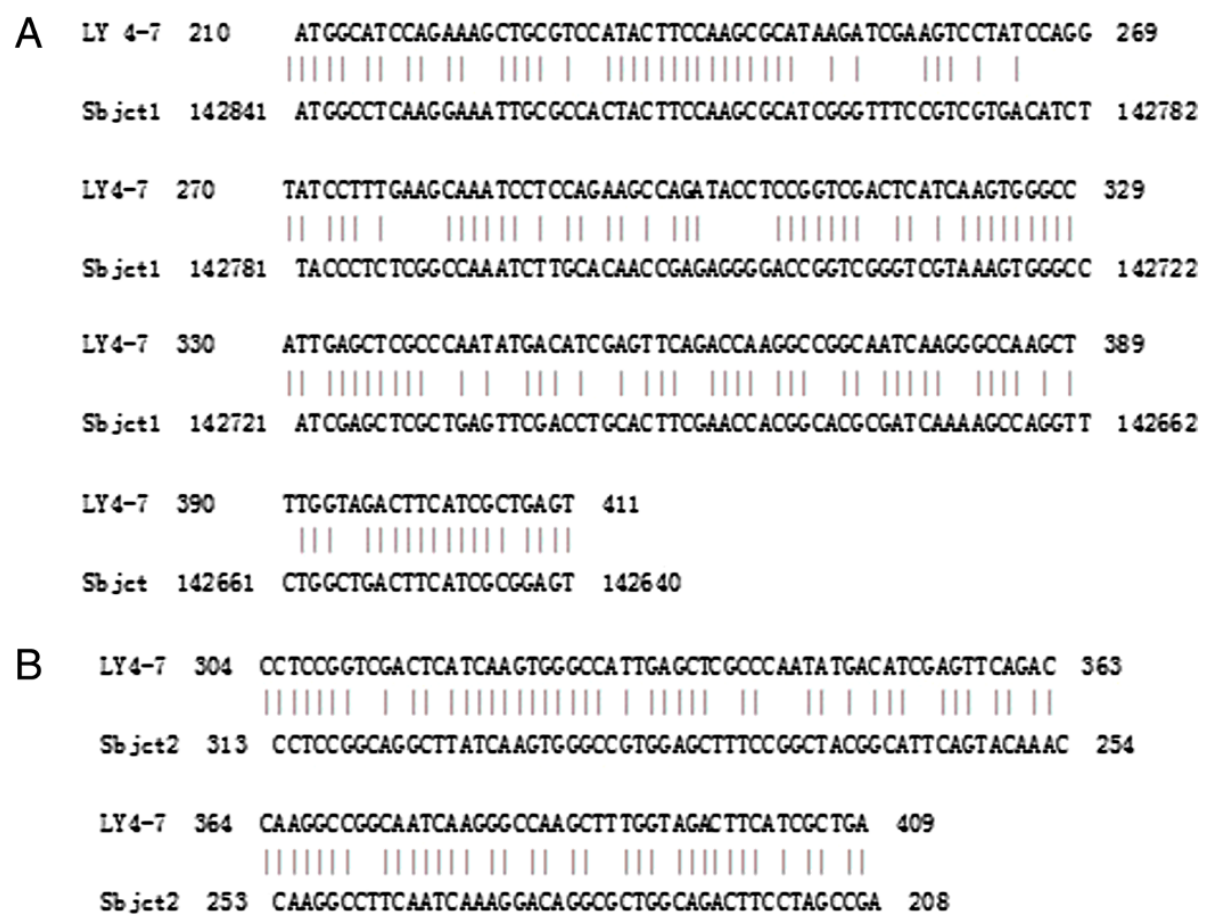

Figure 3 BLAST searches of the cloned nucleotide sequences. Showed identity of clone 4-7 with the Oryza sativa Japonica Group chromosome 10 clone (A), and with the Silene vulgaris isolate $19 \mathrm{~J} 19$ retrotransposon putative Retand type sequence (B).

adopted to find white colony firstly. Then presence of the appropriate insert was verified by PCR with T7/SP6 primer pairs (T7 primer: $5^{\prime}$ - TAA TACGACTCACTATA GGG -3', SP6 primer: 5' -ATTTAGGTGACACTATAGA A-3'), or EcoRI digestion, which is located at pGM-T vector nearly to the ligation ends, and then for DNA sequencing ( $\mathrm{Fu}, 2012)$.

Sequence homology searches and bio-informatics analysis Homology searches were performed by online program BLAST from NCBI (http://www.ncbi.nlm.nih.gov/BLAST/) in different species.

\section{SCAR analysis}

The nucleotide sequence of each of the cloned RAPD fragment was used to design pairs of SCAR primers using Primer 3 software from the website (http://bioinfo. ut.ee/primer3-0.4.0/primer3/). Sequences of the SCAR primers, amplification length and PCR condition were shown

in Table 1. Eighteen of DNA samples, including eight of Canavium album strains, five of $D$. longan varieties LZ, GD, GX, HN, FJ and other 5 kinds of species, which were Viola. philippica (DD), Penthorum sedoides (GJT), Penthorum Chinese (GHC), represents Lonicera japonica (JYH), Gastrodia elata (TM), were used as templates for

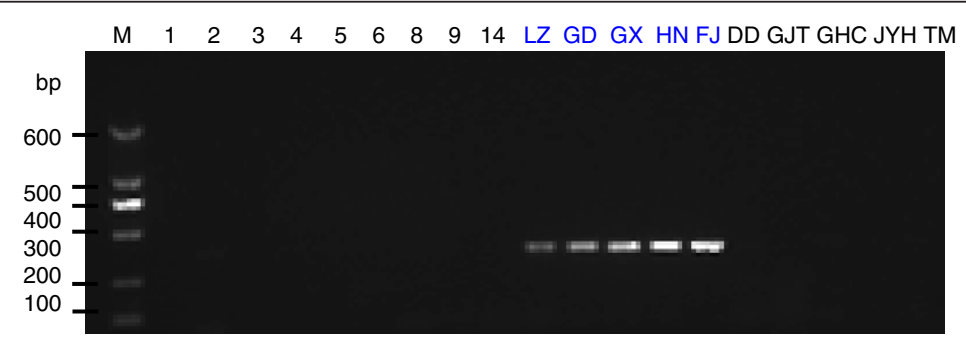

Figure 4 Analysis of the PCR amplicons of a SCAR marker LY2-1. 1, 2, 3, 4, 5, 6, 8, 9 and 14 are C. album samples collected from Sichuan Province. The samples LZ, GD, GX, HN, FJ were collected from Luzhou in Sichuan, Guangdong, Guangxi, Hainan and Fujian sources of D. longan varieties. DD is V. philippica. GJT is P. sedoides. GHC is P. Chinese collected from Gulin County in Sichuan Province. JYH is L. Japonica; TM is Gastrodia elata collected from Liangshan City in Sichuan Province. Lane " $M$ " indicates the DNA molecular weight marker DL600 with the fragment size (bp) 600, 500, 400, 300, 200 and 100 . 


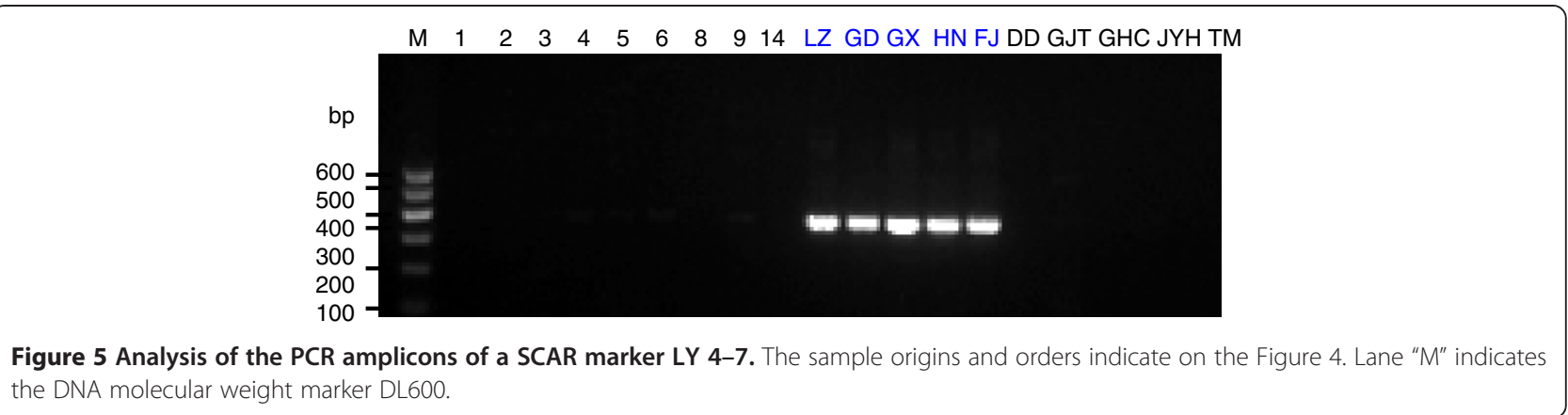

PCR amplification and development of SCAR markers. The PCR reaction solution consisted of $7.5 \mu \mathrm{l} 2 \times$ Taq PCR MasterMix, $1.5 \mu \mathrm{l}$ of $2.5 \mu \mathrm{M}$ each pair of SCAR primers and $1.5 \mu \mathrm{l}$ genomic DNA (15 ng), to a total volume of $15 \mu \mathrm{l}$. Amplification reactions were performed in an Eppendorf Authorized Thermal cycler with an initial pre-denaturation for $90 \mathrm{~s}$ at $95^{\circ} \mathrm{C}$ followed by 35 cycles of denaturation at $94^{\circ} \mathrm{C}$ for $40 \mathrm{~s}$, annealing at $60^{\circ} \mathrm{C}$ for $30 \mathrm{~s}$, and extension at $72^{\circ} \mathrm{C}$ for $40 \mathrm{~s}$. The final extension step was performed at $72^{\circ} \mathrm{C}$ for $5 \mathrm{~min}$. The amplified PCR products were resolved by electrophoresis on $1.0 \%$ agarose gel in $1 \times$ TAE buffer. Gels were visualized by $0.5 \mu \mathrm{g} / \mathrm{mL}$ ethidium bromide staining and the images were documented using the ChemiDoc XRS (Bio-Rad, USA).

To distinguish the difference between $D$. longan and D. confinis, PCR amplifications were performed by using above mentioned 4 pairs of SCAR primers and amplification conditions (Table 1).

\section{Results}

\section{Cloning of RAPD amplification fragments}

Three RAPD primers (SBS-I4, SBS-Q12, and SBS-Q19) were initially screened using DNA samples from Guangxi D. longan (Mei et al. 2013). The characteristic DNA fragments with clear and polymorphic profiles were purified, ligated to T-vector by AT cloning. The blue and white screening method was adopted at first to screen positive cloning. The positive clones $2-1,4-7$, $4-8,5-2$ and 5-4 were identified by PCR amplification using SP6 primer and T7 primer (Figure $1 \mathrm{~A}$ and $\mathrm{B}$ ) or by plasmid DNA digestion using EcoRI enzyme (Figure 1C). In the Figure $1 \mathrm{~A}$, five clones are positive, but only clone 2-1 showed strongest PCR band with a similar inserted fragment and was selected for further sequencing, whereas in the Figure 1B, clones $4-4,4-7$ and 4-8 are PCR positive, and 4-7 and 4-8 with inserted fragments $\sim 600, \sim 900$ bp DNA-fragment respectively. In the Figure $1 \mathrm{C}$, clones 5-2 and 5-4 with a same $\sim 500 \mathrm{bp}$ inserted fragment digested by EcoRI, and clone 5-2 was selected for further sequencing.

\section{Sequencing and characterization of $D$. longan-specific RAPD fragments}

Sequencing of above four cloned RAPD fragments in $D$. longan showed that clone 2-1 consisted of 486 nucleotides and deposited into GenBank with accession number KC522607 (Figure 2A), clone 5-2 consisted of 486 nucleotides and deposited into GenBank with accession number KC522608 (Figure 2B), clone 4-7 consisted of 556 nucleotides and deposited into GenBank with accession number KC522609 (Figure 2C), clone 4-8 consisted of 903 nucleotides and deposited into GenBank with accession number KC522610 (Figure 2D) (Note: the sequences information will not release till one year deposition in Genbank).

BLAST searches of the nucleotide sequences in GenBank showed that 202 nucleotides of clone 4-7 fragment (nucleotides 210 to 411) shared $67 \%$ identity to the Oryza sativa Japonica Group chromosome 10 clone OSJNBa0095J15 sequence (Sequence ID: gb|AC092173.3|) with an E value $8 \mathrm{e}-08$ (Figure $3 \mathrm{~A}$ ), and 78 nucleotides in the same region (nucleotides 304 to 409 ) shared $74 \%$ identity

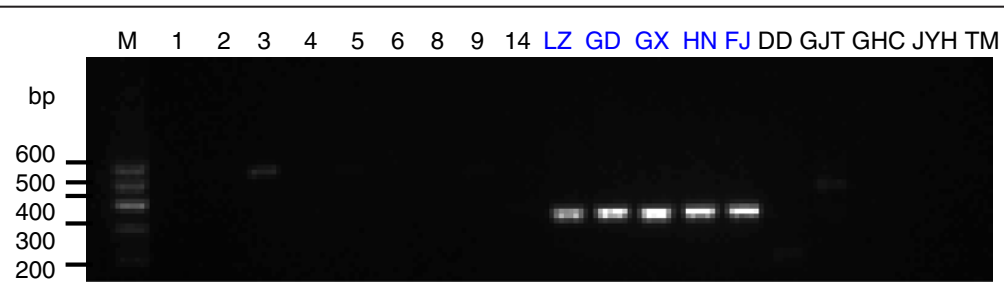

Figure 6 Analysis of the PCR amplicons of a SCAR marker LY 4-8. The sample origins and orders indicate on the Figure 4. Lane "M" indicates the DNA molecular weight marker DL600. 


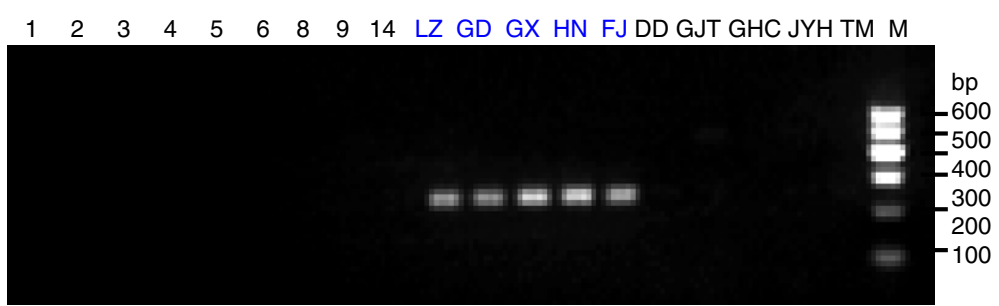

Figure 7 Analysis of the PCR amplicons of a SCAR marker LY 5-2. The sample origins and orders indicate on the Figure 4. Lane "M" indicates the DNA molecular weight marker DL600.

to the Silene vulgaris isolate 19 J19 retrotransposon putative Retand type sequence (Sequence ID: gb| JN624421.1|), with an E value 3e-07 (Figure 3B). Sequences from other 3 clones didn't show any significant identity or similarity to any species.

\section{Development of $D$. longan-specific SCAR markers}

To generate stable longan-specific diagnostic SCAR markers from RAPD markers, four pairs of primers (LY2-1 L and LY2-1R; LY4-7 L and LY4-7R; LY4-8 L and LY4-8R; LY5-2 L and LY5-2R) (Table 1) were designed and synthesized based on cloned sequences in Figure 2. The designed SCAR primer pairs were then used to amplify the genomic DNA from 18 of collected DNA samples to test the amplification species-specificity. PCR results indicated that the PCR products with expected size were observed only in five $D$. longan samples by SCAR marker LY2-1 (Figure 4), SCAR marker LY4-7 (Figure 5), SCAR marker LY4-8 (Figure 6), and SCAR marker LY5-2 (Figure 7), without any amplification in other species we tested (Figures 4,5,6,7), which indicated that all four SCAR markers are longan-specific. The lack of this specific amplicon in the other species indicated the efficacy of these marker in distinguishing the longan group from the others.

\section{Authentication of $D$. longan from $D$. confinis}

To distinguish $D$. longan from $D$. confinis, PCR amplification were performed by using our developed four pairs of SCAR primers (Table 1) with conditions mentioned in Material and Methods. The result showed that the PCR products with expected size were observed only in five

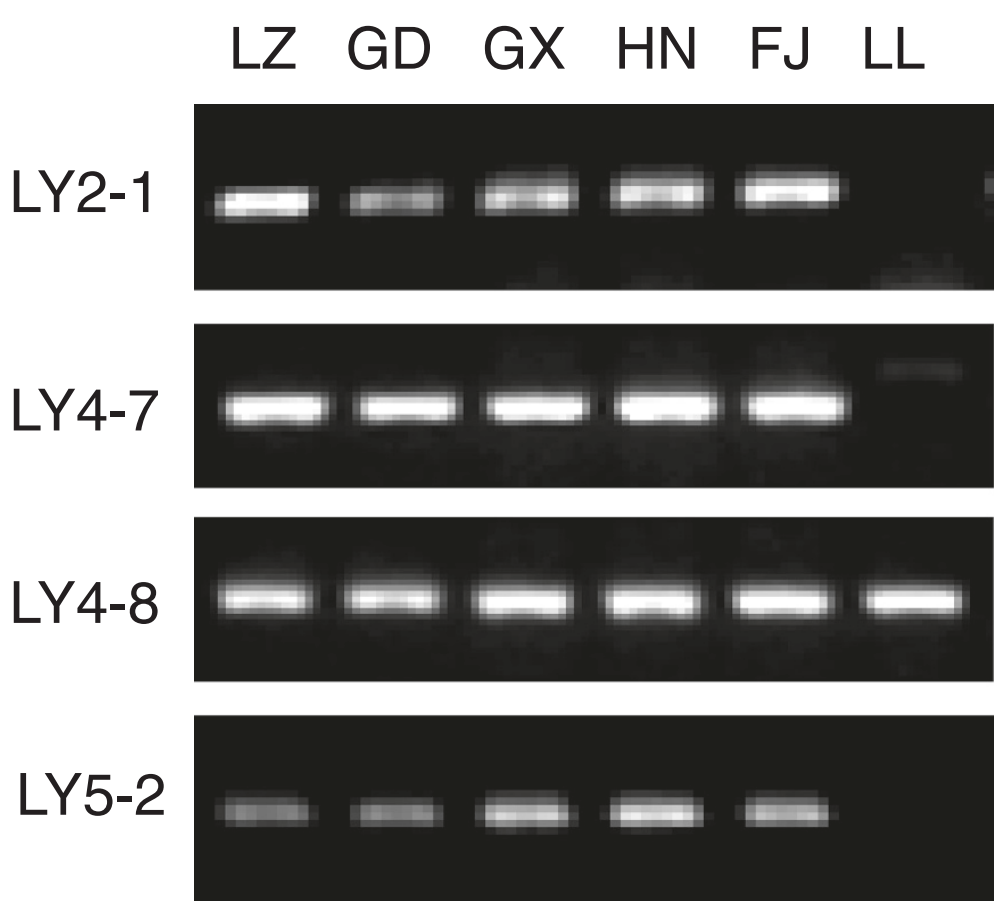

Figure 8 Identification of $D$. longan from $\boldsymbol{D}$. confinis by longan-specific SCAR markers. The samples LZ, GD, GX, HN, FJ were collected from Luzhou in Sichuan, Guangdong, Guangxi, Hainan and Fujian sources of D. longan. The sample LL was collected from Guangxi source of wild D. confinis. LY2-1, LY4-7, LY4-8 and LY5-2 represent longan-specific SCAR markers for LY2-1, LY4-7, LY4-8 and LY5-2 which were used in the PCR amplification, respectively. 
D. longan samples by SCAR markers LY2-1, LY4-7 and LY5-2, without any amplification in the sample of $D$. confinis, which indicated that these three makers are useful for the identification $D$. longan from $D$. confinis (Figure 8 ). However, we still noticed the PCR product in $D$. confinis (LL) with same expected size from $D$. longan by SCAR marker LY4-8, which indicated that this marker can't be used in the authentication of $D$. longan from D. confinis (Figure 8).

\section{Discussion}

RAPD analysis can reveal high degrees of polymorphisms, does not require prior DNA sequence information of the species, and is easy to manipulate (Williams et al. 1990; Devaiah and Venkatasubramanian, 2008; Chen et al. 2010; Yazbeck et al. 2011; Bhat et al. 2012; Shakeel et al. 2013; Noormohammadi et al. 2013; Zhang et al. 2013). Therefore, researchers could explore its application for authentication of traditional Chinese medicines. RAPD needs less DNA template and is relatively easy to handle, but is poor in reproducibility and stability, leading to restrictions in practical application. However, after converting RAPD markers into SCAR markers, the specificity and stability can be greatly improved, which makes it more convenient and efficient in the testing of different alleles (Dnyaneshwar et al. 2006; Li et al. 2010; Rajesh et al. 2013). Since they can identify a single or a few bands instead of a complex pattern, SCAR markers are more straightforward than other molecular techniques, such as RAPD, SSR, ISSR and AFLP.

In this study, we selected the clear and bright amplified DNA bands from RAPD markers for SCAR marker development. In SCAR, pairs of 18-25 bp oligonucleotide primers specific to the sequence of polymorphic bands can be used to amplify the characterized regions from genomic DNA under stringent conditions, which makes these markers more specific and dependable as compared to RAPD markers. Based on the sequences of our cloned DNA fragments, four SCAR primer pairs (LY2-1, LY4-7, LY4-8 and LY5-2) were designed. Genomic D. longan DNAs collected from 5 different regions within China, contained the cloned DNA fragments. As shown in Figures 4,5,6,7, the primers were generated from $250-360$ bp bands in all $D$. longan cultivars, while no amplicon was observed in other species. Thus, the genetic polymorphism observed among the cultivars is interesting and can be used to develop markers for $D$. longan-specific identification.

Traditionally, $D$. longan fruit is used for several diseases (Park et al. 2010; Prasad et al. 2010; Zhong et al. 2010; Mei et al. 2013). Recently it was found that the dried longan seed extracts also have potential inhibitory effects on cancer cell invasion (Panyathep et al. 2013). However, D. confinis, a species of plant in the genus Dimocarpus family, with an oval-shaped drupe fruit similar to D. longan
(http://www.people.com.cn/GB/paper503/13144/1179090. html), is very hard to distinguish from $D$. longan in the market only by morphology. If people sell $D$. confinis as longan, and someone purchase mistakenly and eat, they will experience vomiting, diarrhea and psychiatric disorder including anxiety, depression, insomnia, apprehension, auditory and visual hallucination, and torpid reaction. To distinguish the D. longan and D.confinis, PCR amplifications were performed by using above mentioned SCAR primers from Table 1. The results showed that the PCR products with expected size were observed only in $D$. longan samples by SCAR markers LY2-1, LY4-7 and LY5-2, without any amplification in D. confinis (Figure 8), which suggests that we have developed RAPD-SCAR markers successfully for identification of $D$. longan from the other species with similar morphology. Although the PCR product in $D$. confinis (LL) with same expected size from $D$. longan samples by SCAR marker Y4-8 were detected, which indicates that this marker cannot be used in the identification of $D$. longan from $D$. confinis, we can demonstrate that the DNA quality extracted from $D$. confinis is excellent. Therefore, our results showed that the developed SCAR markers could be useful to assess the genetic diversity and population structure of $D$. longan from $D$. confinis.

In this study, we obtained four clones by improved RAPD and DNA sequencing in D. longan, and developed stably diagnostic Sequence-Characterized Amplified Region (SCAR) markers for DNA fingerprinting to distinguish the fruit of $D$. longan, as a species of plant in the genus Dimocarpus with similarity to that of $D$. confinis with poisonous nature. Our results have shown that these SCAR markers could be very useful as a specific $D$. longan variety authentication, particularly for distinguishing $D$. longan from $D$. confinis.

\section{Competing interests}

The authors declare that they have no competing interests.

\section{Authors' contributions}

LY performed major experiments. SF and MAK performed experimental assistance and helped in revision of the manuscript. WZ designed the project and helped in preparation of the manuscript. JF designed the project \& experiments, wrote the first draft of the manuscript. All authors read and approved the final manuscript.

\section{Acknowledgments}

This research was supported in part by the National Natural Science Foundation of China (30371493 and 81172049), and the Science and Technology Innovation Team of Colleges and Universities in Sichuan Province (13TD0032). The authors particularly thank all individuals who provided DNAs or leaves for D. longan, D. confinis and other species.

\section{Author details}

${ }^{1}$ Research Center for Preclinical Medicine, Luzhou Medical College, Luzhou, Sichuan 646000, China. ${ }^{2}$ Michael E. DeBakey High School for Health Professions, 3100 Shenandoah Street, Houston, TX 77021, USA. ${ }^{3}$ Department of Biochemistry, School of Life Sciences, Central South University, Changsha, Hunan 410013, China. 
Received: 9 August 2013 Accepted: 18 September 2013

Published: 3 October 2013

\section{References}

Bhat AA, Haniffa MA, Divya PR, Gopalakrishnan A, Milton MJ, Kumar R, et al. (2012) Molecular characterization of eight Indian Snakehead species (Pisces: Perciformes Channidae) using RAPD markers. Mol Biol Rep 39:4267-4273

Chen H, He X, Pan J, Zhu J (2010) Advances on Application of Molecular Markers in Longan Researches. Chinese Agric Sci Bul 26:26-30

Devaiah KM, Venkatasubramanian P (2008) Genetic characterization and Panyathepauthentication of Embelia ribes using RAPD-PCR and SCAR marker. Planta Med 74:194-196

Dnyaneshwar W, Preeti C, Kalpana J, Bhushan P (2006) Development and application of RAPD-SCAR marker for identification of Phyllanthus emblica LINN. Biol Pharm Bull 29:2313-2316

Feofilov AV, Bardukov NV, Glazko VI (2011) Gene pool differentiation between Altaic and trotting horse breeds inferred from ISSR-PCR marker data. Genetika 47:1230-1235

Fu JJ (2012) Short protocols in medical molecular biology. China Medical Science Press, Beijing

Fu J, Li L, Xu X, Wang Z, Tang G, Yin C, Lu G (2000) An improved method for increasing the efficiency of the technique of random amplified polymorphic DNA (RAPD). Hereditas 22:251-252

Fu J, Yang L, Khan MA, Mei Z (2013) Genetic characterization and authentication of Lonicera japonica Thunb. by using improved RAPD analysis. Mol Biol Rep. 10.1007/s11033-013-2703-3

Ganopoulos I, Merkouropoulos G, Pantazis S, Tsipouridis C, Tsaftaris A (2011) Assessing molecular and morpho-agronomical diversity and identification of ISSR markers associated with fruit traits in quince (Cydonia oblonga). Genet Mol Res 10:2729-2746

Kumla S, Doolgindachbaporn S, Sudmoon R, Sattayasai N (2012) Genetic variation, population structure and identification of yellow catfish, Mystus nemurus (C\&V) in Thailand using RAPD, ISSR and SCAR marker. Mol Biol Rep 39:5201-5210

Li SF, Tang SJ, Cai WQ (2010) RAPD-SCAR markers for genetically improved NEW GIFT Nile Tilapia (Oreochrmis niloticus niloticus L.) and their application in strain identification. Zool Res 31:147-153

Mei Z, Fu S, Yu H, Yang L, Duan C, Liu X, Gong S, Fu J (2013) Genetic characterization and authentication of Dimocarpus longan Lour. using an improved RAPD technique. Genet Mol Res 12. in press

Micheli MR, Bova R, Pascale E, Ambrosio E (1994) Reproducible DNA fingerprint with the random amplified polymorphic DNA (RAPD) method. Nucleic Acids Res 22:1921-1922

Noormohammadi Z, Hasheminejad-Ahangarani FY, Sheidai M, GhasemzadehBaraki AS, Alishah O (2013) Genetic diversity analysis in Opal cotton hybrids based on SSR, ISSR, and RAPD markers. Genet Mol Res 12:256-269

Panyathep A, Chewonarin T, Taneyhill K, Vinitketkumnuen U, Surh YJ (2013) Inhibitory effects of dried Longan ( Euphoria longana Lam.) seed extract on invasion and matrix metalloproteinases of colon cancer cells. J Agric Food Chem 61:3631-3641

Park SJ, Park DH, Kim DH, Lee S, Yoon BH, Jung WY, et al. (2010) The memory-enhancing effects of Euphoria longan fruit extract in mice. J Ethnopharmacol 128:160-165

Prasad KN, Yang B, Shi J, Yu C, Zhao M, Xue S, et al. (2010) Enhanced antioxidant and antityrosinase activities of longan fruit pericarp by ultra-high-pressure-assisted extraction. J Pharm Biomed Anal 51:471-477

Rajesh MK, Jerard BA, Preethi P, Thomas RJ, Fayas TP, Rachana KE, et al. (2013) Development of a RAPD-derived SCAR marker associated with tall-type palm trait in coconut. Sci Hortic 150:312-316

Shakeel M, Ilyas M, Kazi M (2013) Evaluation of synthetic hexaploid wheats (derivative of durum wheats and Aegilops tauschii accessions) for studying genetic diversity using randomly amplified polymorphic DNA (RAPD) markers. Mol Biol Rep 40:21-26

Varela ES, Limam JP, Galdino AS, Pinto Lda S, Bezerra WM, Nunes EP, et al. (2004) Relationships in subtribe Diocleinae (Leguminosae; Papilionoideae) inferred from internal transcribed spacer sequences from nuclear ribosomal DNA. Phytochemistry 65:59-69

Vos P, Hogers R, Bleeker M, Reijans M, van de-Lee T, Hornes M, et al. (1995) AFLP: a new technique for DNA fingerprinting. Nucleic Acids Res 23:4407-4414
Williams JG, Kubelik AR, Livak KJ, Rafalski JA, Tingey SV (1990) DNA polymorphisms amplified by arbitrary primers are useful as genetic markers. Nucleic Acids Res 18:6531-6535

Yazbeck GM, Brandão RL, Cunha HM, Paglia AP (2011) Detection of two morphologically cryptic species from the cursor complex (Akodon spp; Rodentia, Cricetidae) through the use of RAPD markers. Genet Mol Res 10:2881-2892

Zhang L, Zhang HG, Li XF (2013) Analysis of genetic diversity in Larix gmelinii (Pinaceae) with RAPD and ISSR markers. Genet Mol Res 12:196-207

Zhong K, Wang Q, He Y, He X (2010) Evaluation of radicals scavenging, immunity-modulatory and antitumor activities of longan polysaccharides with ultrasonic extraction on in S180 tumor mice models. Int J Biol Macromol 47:356-360

doi:10.1186/2193-1801-2-501

Cite this article as: Yang et al:: Molecular cloning and development of RAPD-SCAR markers for Dimocarpus longan variety authentication. SpringerPlus 2013 2:501.

\section{Submit your manuscript to a SpringerOpen ${ }^{\circ}$ journal and benefit from:}

- Convenient online submission

Rigorous peer review

- Immediate publication on acceptance

- Open access: articles freely available online

- High visibility within the field

- Retaining the copyright to your article

Submit your next manuscript at springeropen.com 